\title{
Algebraic and geometric space-time analogies in nonlinear optical pulse propagation
}

\author{
Shayan Mookherjea and Amnon Yariv \\ Department of Electrical Engineering, 136-93 California Institute of Technology, 1200 East California Boulevard, \\ Pasadena, California 91125
}

Received March 13, 2001

\begin{abstract}
We extend recently developed algebraic space-time analogies for the dispersive and nonlinear propagation of optical breathers. Geometrical arguments can explain the similarity of evolutionary behavior between spatial and temporal phenomena even when strict algebraic translation of solutions may not be possible. This explanation offers a new set of tools for understanding and predicting the evolutionary structure of self-consistent Gaussian breathers in nonlinear optical fibers. (c) 2001 Optical Society of America

OCIS codes: $060.2330,060.5530,060.4370$.
\end{abstract}

Analogies between spatial Gaussian beam diffraction and temporal Gaussian pulse dispersion have been well established ${ }^{1}$ and were extended more recently to introduce the concept of a time lens as a quadratic phase modulator, applied in temporal imaging configurations, for temporal filtering and transforms $\mathrm{s}^{2,3}$ and in nonlinear dispersive optical communications. $^{4,5}$ Here we investigate Gaussian optical pulse propagation with periodically varying pulse width and chirp ("breathers") in a nonlinear dispersive fiber in the context of such space-time analogies but from a topological perspective rather than the algebraic correspondence of variables. This method, illustrated here in the simplest of cases, can lead to a deeper understanding of pulse propagation based on the geometrical structure of the topological space of solutions and their classifications in terms of homotopy groups.

The problem of pulse propagation in a dispersive nonlinear medium has basic features that are strongly suggestive of steady-state optical beam propagation in a medium with a quadratic radial index dependence. That this is so can be seen, for instance, in Fig. 1, which shows the results of numerical simulations of [Fig. 1(a)] Gaussian pulse evolution in a hypothetical optical fiber described such that the propagation equations are exactly analogous to those describing Gaussian beam evolution in a rotationally symmetric quadratic graded-index (RSQ-GRIN) medium and [Fig. 1(b)] Gaussian pulse evolution in an optical fiber with the Kerr effect. Since, as discussed below, closed-form spatially periodic solutions to the earlier problem [Fig. 1(a)] are known, it is intriguing to inquire whether the same form of the solution can be applied to the second problem [Fig. 1(b)], which is of practical interest, and if not, where the analogy fails despite the easily visible affinity shown in Fig. 1.

We study this problem in the context of space-time dualities by first looking at the spatial beam-diffraction problem. For plane waves with a Gaussian envelope $A(r, z)$ propagating with complex propagation constant $k(r)$ in a RSQ-GRIN medium characterized by quadratic constant $k_{2},{ }^{6}$ Maxwell's equations and the slowly varying envelope approximation lead to a parabolic partial differential equation for $A(r, z)$ :

$$
\nabla_{\perp}^{2} A-2 i k \frac{\partial A}{\partial z}-k k_{2} r^{2} A=0,
$$

where $\nabla_{\perp}^{2}$ is the transverse Laplacian operator and $r$ is the distance from the cylindrical axis of symmetry. We typically use the following form for the envelope ${ }^{6}$ :

$$
A(r, z)=\exp \left\{i\left[P(z)+\frac{k}{2 q(z)} r^{2}\right]\right\}
$$

with the $q$ parameter

$$
\frac{1}{q(z)} \equiv \frac{1}{R(z)}-i \frac{2 \lambda}{k w^{2}(z)}
$$

defined in terms of the beam spot size, $w(z)$, and the radius of curvature of the beam, $R(z)$. The solution $q(z)$ for a given unchirped $[R(0)=0]$ input characterized by $q(0)=q_{0}$ is expressed in closed form by an $A B C D$ bilinear transformation ${ }^{6}$ :

$$
q(z)=\frac{A(z) q_{0}+B(z)}{C(z) q_{0}+D(z)}
$$

where

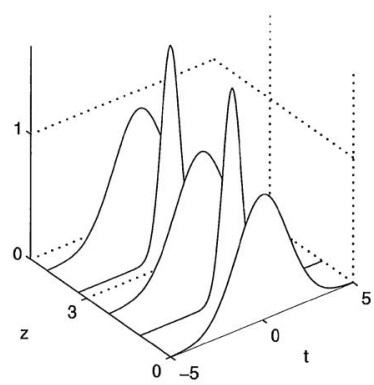

(a)

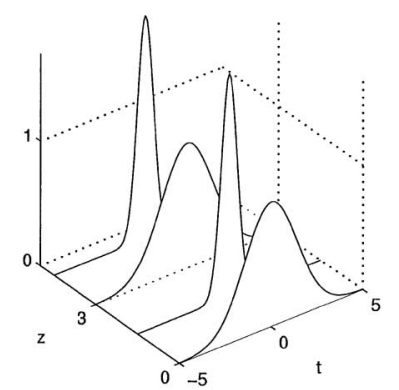

(b)
Fig. 1. Temporal evolution of a Gaussian breather pulse (a) in a hypothetical medium described by Eq. (7) and exactly analogous to Gaussian beam propagation in a RSQ-GRIN fiber and (b) in a realistic fiber with the Kerr effect, as described by Eq. (10). 


$$
\begin{aligned}
& A(z)=\cos \left(\sqrt{k_{2} / k} z\right), \quad B(z)=\sqrt{k / k_{2}} \sin \left(\sqrt{k_{2} / k} z\right), \\
& C(z)=-\sqrt{k_{2} / k} \sin \left(\sqrt{k_{2} / k} z\right), \quad D(z)=\cos \left(\sqrt{k_{2} / k} z\right) .
\end{aligned}
$$

This form of the solution clearly shows the breathing nature of the wave envelope, i.e., periodic in width and radius of curvature.

Next, in discussing temporal Gaussian pulse propagation, we know that group-velocity dispersion leads to broadening or narrowing of the temporal envelope, depending on the signs of the group-velocity dispersion parameter, $\beta^{\prime \prime}$, and the chirp, although the shape of the envelope remains Gaussian. We may counteract this effect by utilizing self-phase modulation caused by a nonzero nonlinear-index coefficient $n_{2}$. Breathers can be formed when spectral broadening from positive $n_{2}$ balances anomalous dispersion $\left(\beta^{\prime \prime}<0\right)$ in fibers. Similarly, negative $n_{2}$ as found in AlGaAs waveguides ${ }^{7}$ can compensate for the effect of normal dispersion, and breathers can be created in this case as well. Self-consistent Gaussian breathers differ from conventional solitons ${ }^{6}$ in that the pulse parameters vary periodically such that the pulse envelope can, by assumption, always be described by a Gaussian function.

If self-phase modulation were to result in a (temporal) term analogous to the $k k_{2} r^{2}$ term in Eq. (1), the solution would be temporally breathing Gaussian pulses, similar to the spatial case of breathing Gaussian beams, and this would satisfactorily explain the similarities of Figs. 1(a) and 1(b): The solution would be given in closed form by the temporal equivalent of Eq. (4), by use of the space-time translation rules $^{4,5}$

$$
\begin{gathered}
z \mapsto z, \quad r \mapsto T, \quad \nabla_{\perp}^{2} \mapsto \nabla_{T}^{2}, \\
k \mapsto-1 / \beta^{\prime \prime}, \quad k_{2} \mapsto 4 \gamma,
\end{gathered}
$$

where $T=t-z / v_{g}$ is the temporal coordinate in the reference frame that is comoving with the pulse at the group velocity and $\gamma=n_{2} \omega_{0} /\left(c A_{\text {eff }}\right)$ is the nonlinearity coefficient defined in terms of optical frequency $\omega_{0}$ and effective core area $A_{\text {eff }}$.

It can be easily seen, however, that such is not the case. Applying the translation rules (6) to Eq. (1), we obtain

$$
-i \frac{\partial A}{\partial z}+\frac{\beta^{\prime \prime}}{2} \frac{\partial^{2} A}{\partial T^{2}}+\frac{b_{2}}{2} T^{2} A=0
$$

which we refer to as the temporal RSQ-GRIN problem; its solution can be written explicitly in the $A B C D$ form. But the wave equation governing the propagation of the envelope of a Gaussian pulse in a nonlinear dispersive fiber is ${ }^{6}$

$$
-i \frac{\partial A}{\partial z}+\frac{\beta^{\prime \prime}}{2} \frac{\partial^{2} A}{\partial T^{2}}-\gamma \frac{\Delta n}{n^{2}} A=0,
$$

where $n_{2}$ is the (constant) nonlinear index of refraction and $\Delta n(z, T)$ is the Kerr-effect field-induced change in the refractive index. For a Gaussian pulse envelope of width $\tau(z), \Delta n$ can be approximated as

$$
\Delta n(z, T)=n_{2} \exp \left[-2 \frac{T^{2}}{\tau^{2}(z)}\right] \approx n_{2}\left[1-\frac{2 T^{2}}{\tau^{2}(z)}\right]
$$

where we assume that the nonlinearity responds instantaneously. Equation (8) becomes

$$
-i \frac{\partial A}{\partial z}+\frac{\beta^{\prime \prime}}{2} \frac{\partial^{2} A}{\partial T^{2}}-\gamma\left[1-\frac{2 T^{2}}{\tau^{2}(z)}\right] A=0
$$

and, clearly, Eqs. (7) and (10) are not identical. Therefore, the $A B C D$ form of the solution cannot be applied to Eq. (10).

To explain the similarity shown in Figs. 1(a) and 1(b), we seek a different approach. To identify analogies with the analysis of the spatial RSQ-GRIN problem, Eq. (2), we write the temporal-pulse Ansatz as

$$
A(z, T)=\exp \left\{i\left[P(z)+\frac{T^{2}}{2 \beta^{\prime \prime} q(z)}\right]\right\},
$$

with a temporal $q$ parameter,

$$
\begin{aligned}
\frac{1}{q(z)} & \equiv \frac{1}{R(z)}+i \frac{2 \beta^{\prime \prime}}{\tau^{2}(z)} \\
& \equiv r(z)[\cos \theta(z)+i \sin \theta(z)],
\end{aligned}
$$

where $R(z)$ describes the chirp of the pulse and $\tau(z)$ is its width. (We use the polar representation in our analysis below). The chirped Gaussian envelope that this formalism describes may, for instance, be one member of a complete orthonormal basis of Hermite-Gaussian functions.

We can simplify each of the partial differential equations [Eqs. (7) and (10)] into a pair of ordinary differential equations for $P(z)$ and $q(z)$ by use of Eq. (11) and then equating the coefficients of different powers of $T$. We know from Gaussian beam diffraction analy$\operatorname{sis}^{6}$ that the equation with terms that are quadratic in $T$ involves only the initial-value problem for $q(z)$. As $q(z)$ is complex, it is simplest to carry out a phase-plane analysis in each of the two systems in polar $(r, \theta)$ coordinates by use of the definition presented in Eq. (12). In polar coordinates, we can graph and compare the evolution of the solutions in the $r-\theta$ phase plane, with $z$ as the implicit variable driving the evolution of the instantaneous solution along one of the contours in the phase plane.

The temporal RSQ-GRIN problem [Eq. (7)] can be written in polar coordinates as

$$
\begin{aligned}
\mathrm{d} r / \mathrm{d} z & =r^{2} \cos \theta+2 \gamma \cos \theta \\
\mathrm{d} \theta / \mathrm{d} z & =\frac{1}{r}\left(r^{2} \sin \theta-2 \gamma \sin \theta\right) \\
r(0) & \equiv r_{0}, \quad \theta(0) \equiv \theta_{0}=\pi / 2
\end{aligned}
$$

and for the equations describing pulse propagation in a nonlinear dispersive fiber Eq. (10), 


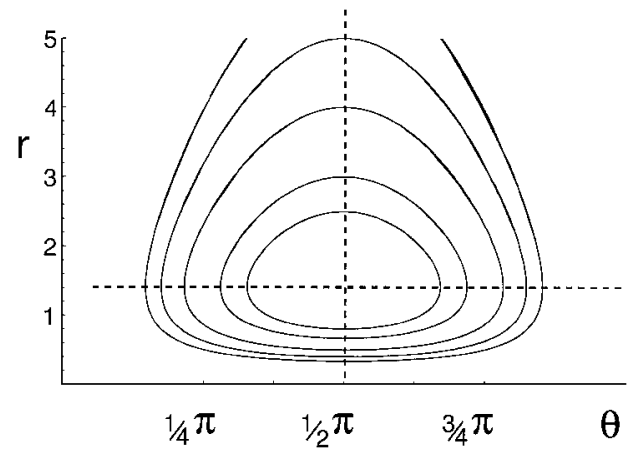

Fig. 2. Temporal evolution of a Gaussian breather in the (hypothetical) temporal RSQ-GRIN medium: phase-plane analysis of Eq. (13).

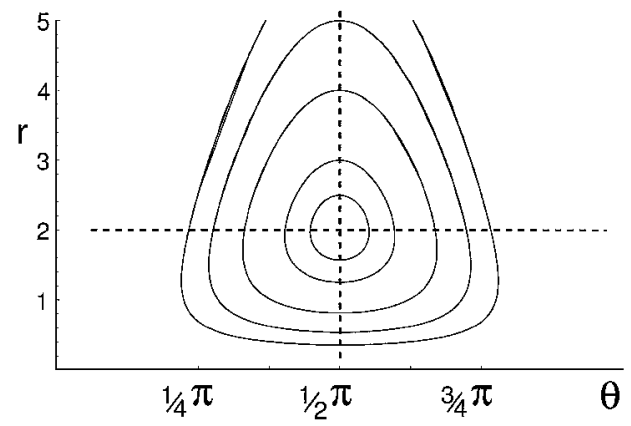

Fig. 3. Temporal evolution of a Gaussian breather in the presence of the Kerr effect: phase-plane analysis of Eq. (14).

$$
\begin{aligned}
\mathrm{d} r / \mathrm{d} z & =r^{2} \cos \theta+2 \gamma \cos \theta(r \sin \theta), \\
\mathrm{d} \theta / \mathrm{d} z & =\frac{1}{r}\left[r^{2} \sin \theta-2 \gamma \sin \theta(r \sin \theta)\right], \\
r(0) & \equiv r_{0}, \quad \theta(0) \equiv \theta_{0}=\pi / 2 .
\end{aligned}
$$

The phase-plane analysis, shown in Figs. 2 and 3 for a particular set of orbits with the same initial conditions, shows the essential difference between Eqs. (13) and (14): Note that in the regime of interest the multiplicative term $r \sin \theta$, which appears in Eq. (14) but not in Eq. (13), is always positive. The evolution of the temporal RSQ-GRIN solution to Eq. (13) (for which we have a closed-form $A B C D$ expression) is symmetric in the phase plane about $\theta=\pi / 2$. So, the term that changes in $\mathrm{d} r / \mathrm{d} z$ for Eq. (14) results in scaling of the contours outward from the nonlinear center in the phase plane without affecting the symmetry about $\theta=\pi / 2$. Consequently, closed orbits that are the solutions for one equation remain closed for the other, although the fixed points for the two problems are different.

Along similar lines, the difference in the $\mathrm{d} \theta / \mathrm{d} z$ terms maintains the same sign in both Eqs. (13) and (14). If $r \sin \theta<1$, the rate of change of $\theta$ decreases, and the period of the breathing solutions increases, which has been verified by numerical solution of Eqs. (13) and (14).

The orbits in phase space are scaled nonlinearly around a fixed point in a way that preserves the structure of the solution space. Consider the sets of solutions of Eq. (13), $X_{1}=\left\{S_{1}\left(r_{0}, \theta_{0}\right)\right\}$, and Eq. (14), $X_{2}=\left\{S_{2}\left(r_{0}, \theta_{0}\right)\right\}$. We take $\theta_{0} \equiv \pi / 2$ for simplicity. The collection of subsets of $X_{1}$ and $X_{2}$, defined as

$$
\tau_{i}=\left\{S_{i}\left(r_{0}\right) \mid a<r_{0}<b\right\}, \quad \forall a, b \geq 0,
$$

define a topology on $X_{1}$ and $X_{2}$. The orbits of the sets of solutions $X_{1}$ and $X_{2}$ are homotopic, which is an equivalence relation on the set of topological spaces. $^{8}$ In fact, the fundamental groups (first homotopy groups) are isomorphic to $\mathbb{Z}$, the fundamental group of the circle,

$$
\pi_{1}\left(X_{1}\right) \cong \pi_{1}\left(X_{2}\right) \cong \pi_{1}\left(S^{1}\right) \cong \mathbb{Z} .
$$

This isomorphism between the first homotopy groups explains the very close similarity of breathing Gaussian pulses in the case of the Kerr effect and Gaussian beams in a graded-index medium, even though the equations for one are not a direct translation of the other.

If there were a closed-form solution to the problem of Gaussian beam propagation in a rotationally symmetric graded-index medium described by a more general relationship than was assumed in Eq. (1) - for example, involving higher powers of $r^{2}$ — then we could retain further terms in the Taylor series expansion in Eq. (9) and apply the same topological arguments to justify the similarities of the two families of solutions. The practical usefulness of such arguments depends, however, on the existence of closed-form solutions in at least one of the two cases-spatial or temporal-so that the solutions in the other case can be demonstrated to be topologically equivalent or related. We expect that more such examples will be identified in the future, leading to a deeper understanding of space-time analogies in optical propagation.

We acknowledge useful discussions with D. S. Cohen of the Department of Applied and Computational Mathematics at the California Institute of Technology. This work was supported by the U.S. Office of Naval Research and the U.S. Air Force Office of Scientific Research. S. Mookherjea's e-mail address is shayan@caltech.edu.

\section{References}

1. S. Akhmanov, A. Chirkin, K. Drabovich, A. Kovrigin, R. Khokhlov, and A. Sukhorukov, IEEE J. Quantum Electron. QE-4, 598 (1968).

2. B. Kolner and M. Nazarathy, Opt. Lett. 14, 632 (1989).

3. A. Lohmann and D. Mendlovic, Appl. Opt. 31, 6212 (1992).

4. S. Mookherjea and A. Yariv, Phys. Rev. E 64, 016611 (2001).

5. A. Yariv, J. Nonlinear Opt. Phys. Mater. 8, 165 (1999).

6. A. Yariv, Optical Electronics in Modern Communications (Oxford University, New York, 1997).

7. M. LaGasse, K. Anderson, C. Wang, H. Haus, and J. Fujimoto, Appl. Phys. Lett. 56, 417 (1990).

8. M. Nakahara, Geometry, Topology and Physics (Institute of Physics, London, 1990). 